\title{
Notes critiques et documents
}

\section{Nouveaux éclairages sur l'émir Abd el-Kader : la documentation napolitaine*}

\author{
Federico Cresti \\ Université de Catane, Italie
}

1. Les documents des anciens États napolitains, aujourd'hui conservés par l'Archivio di Stato de Naples, constituent l'une des sources les plus riches, en Italie, en ce qui concerne l'histoire des rapport entre le rivage septentrional et le rivage méridional de la Méditerranée, et plus en particulier, pour l'histoire des territoires du Maghreb à l'époque moderne et contemporaine depuis le moyen âge jusqu'à la période de la formation de l'état unitaire italien ${ }^{1}$.

L'importance de ces archives pour les chercheurs intéressés à l'histoire de l'Afrique méditerranéenne a été premièrement révélée par les travaux d'inventaire réalisés au cours des années '6o et '70 du siècle passé : je me bornerai à citer le troisième volume (d'environ 500 pages) de l'inventaire des sources manuscrites italiennes concernant l'histoire de l'Afrique du $\mathrm{Nord}^{2}$, réalisé par une équipe de chercheurs coordonnés par Teobaldo

* Texte de la communication présentée au colloque international : « Le legs de l'émir Abdelkader entre particularisme et universalité : approches analytiques », Université d'Oran, 29-30 novembre 2008.

1 Il suffira ici de rappeler que la formation de l'État unitaire peut se dire achevée en 1870 , lorsque Rome devient la capitale de l'Italie. Dans la décennie précédente, les anciens états qui auparavant gouvernaient la péninsule avaient été absorbés par le royaume d'Italie (proclamé en 1861) : le royaume des Deux Siciles fut annexé en 1860, et ses attributions politiques et administratives furent transférées aux institutions unitaires.

2 Le programme de recherche ayant pour thème l'Inventario delle fonti manoscritte relative alla Storia dell'Africa del Nord esistenti in Italia [Inventaire des 
Filesi et dédié, justement, aux archives de Naples ${ }^{3}$. Plus récemment, d'autres historiens se sont penché sur les documents de ces archives afin d'en rendre plus aisé l'accès aux chercheurs intéressé à tel ou tel autre aspects de l'histoire des pays maghrébins. Je citerai avant tout le travail mené par l'équipe dirigée par Anna Bozzo (dont ont fait partie Luciana Siano, Immacolata Caruso et Stefania Botta) sur les documents concernant l'Algérie dans la période de 1782 à $1862^{4}$ : cet inventaire des archives du ministère des Affaires étrangères du royaume des Deux Siciles a constitué le point de départ de mon travail sur les documents napolitains concernant l'épopée de l'émir Abd el-Kader.

2. Il pourrait paraître surprenant, à première vue, que les archives de Naples conservent une telle quantité de documents relatifs au Maghreb central : moins surprenant, toutefois, si l'on rappelle que les relations économiques et commerciales entre l'Italie méridionale et les côtes maghrébines (bien qu'informelles, le plus souvent, et caractérisées par la violence de la guerre corsaire) avaient été suivies et continues pendant une très longue période.

Au cours du XVIII e siècle le royaume des Deux Siciles avait à plusieurs reprises essayé de normaliser ses rapports avec la régence d'Alger par un traité de paix et de commerce : les gouvernants d'Alger, de leur côté, avaient plusieurs fois refusé les avances napolitaines, surtout en raison du fait que Charles III de Bourbon (qui siégea sur le trône de Naples à partir de 1734 et sur celui d'Espagne à partir de 1759) et sa dynastie étaient les plus âpres ennemis d'Alger. Aucune paix n'aurait été possible avec eux avant la resti-

sources manuscrites pour l'Histoire de l'Afrique du Nord existant en Italie], qui a réuni différentes équipes de chercheurs italiens, a eu parmi ses résultats la publication de plusieurs volumes, dédiés entre autres aux archives de l'ancien ministère des Colonies, du ministère de la Défense, et aux archives ecclésiastiques romaines.

3 T. Filesi (ed.), The public Archives of Campania with special reference to the Archives of State in Naples from the beginning until 1922, Brill, Leiden 1973. Cf. aussi, du même auteur, T. Filesi, Il programma di ricerca, selezione, pubblicazione dei documenti esistenti negli Archivi della Campania e relativi ai rapporti con i Paesi dell'Africa del Nord nei secoli XVIII-XX, in «Africa » [Rome], n. 3/1972, p. 421-427. Un travail de caractère plus général, mais toujours très utile pour comprendre la structure et le contenu des archives napolitaines, est celui de J. Mazzoleni, Le fonti documentarie e bibliografiche dal sec. X al secolo XX conservate presso l'Archivio di Stato di Napoli, I, Arte Tipografica, Napoli 1974.

4 A. Bozzo (sous la direction de), L'Algeria nei documenti dell'Archivio di stato di Napoli. Fondo Affari Esteri (1782-1862), Istituto per l'Oriente, Roma-Napoli 1992. 
tution d'Oran aux algériens ${ }^{5}$. Toutefois, même après l'évacuation d'Oran par les espagnols en 1792 la situation n'avait pas changé.

Seulement en 1813 une trêve avait été signée entre les Bourbons et le dey d'Alger par l'entremise du gouvernement anglais. Des sujets britanniques ${ }^{6}$ furent chargés, dans les années suivantes, de gérer les affaires consulaires de Naples, sans toutefois pouvoir empêcher les attaques des corsaires contre les navires napolitains : en 1815 le consul anglais Hugh Mac Donell fut investi de la charge de consul général du royaume des Deux Siciles auprès de la Régence?

Une véritable normalisation des rapports n'intervint toutefois qu'en 1816, suite à la première démonstration navale de l'amiral Edward Pellew, lord Exmouth, contre la ville : le 3 avril 1816 Exmouth signa, pour le compte aussi du roi de Naples, un traité de paix avec le gouvernement de la Régence qui prévoyait entre autres la libération des sujets napolitains qui se trouvaient en esclavage dans les bagnes de la ville moyennant le paiement d'une rançon ${ }^{8}$. Les intérêts du royaume de Naples dans les territoires du Maghreb central se bornaient à cette époque, outre qu'au rachat des esclaves, aux activités des corallari, c'est-à-dire des pêcheurs de corail qui se déplaçaient périodiquement vers les côtes maghrébines à partir des ports de l'Italie méridionale, de Torre del Greco et de Trapani en particulier:

5 Cf. T. Filesi, Napoli e Algeri tra il 1740 e il 1830, in R.H. Rainero (sous la direction de), Italia e Algeria. Aspetti storici di un'amicizia mediterranea, Marzorati, Milano 1982, p. 97-10o. Des négociations pour établir au moins une trêve avaient été entamées par la cour napolitaine en 1761,1769 et 1773 ; de 1783 à 1787 un traité de paix avait été discuté qui n'avait jamais abouti (ibid., p. 101. Les documents relatifs à cette dernière tentative de paix se trouvent dans les archives napolitaines : Archivio di Stato di Napoli [dorénavant: ASN], Affari Esteri [Affaires Étrangères, dorénavant: $\mathrm{AE}], 4212)$.

6 Moore en 1814, M. Chamberlagne et H. Mac Donell par la suite (T. Filesi, Napoli e Algeri ... cit., p. 120-122).

7 La nomination officielle de Mac Donell est datée du 14 novembre 1815 (T. Filesi, Napoli e Algeri ... cit., p. 126-127). D'après A. Bozzo (op. cit., p. 342, où se trouve la liste des consul napolitains avec les dates de leur nomination) Mac Donell — qui est cité omm Mac Donnell dans d'autres documents — ne fut nommé consul qu'en février 1817 .

8 Le 'traité de paix perpétuelle' entre Ferdinand IV et le dey d'Alger est publié par le «Giornale di Palermo», n. 301, 12.5.1816 (copie in ASN, AE, 2395). Sur les tentatives diplomatiques napolitaines de paix avec Alger, cf. aussi A. Cilento, Il governo delle Due Sicilie alla ricerca di una pace stabile con le Reggenze Barbaresche (1799-1816), in « Africa » [Rome], 1/1969, p. 41-69. 
cette activité maritime, qui était en grande partie l'apanage de sujets napolitains, mettait en jeu d'importantes ressources économiques ${ }^{9}$.

Lors de la deuxième expédition d'Exmouth, qui bombarda la ville le 27 août de la même année, Mac Donell avait été arrêté par ordre du dey et Renato De Martino, consul napolitain à Tunis, avait été nommé vice-consul pour le remplacer ${ }^{10}$. Des considérations de politique internationale avaient vu dans les années suivantes la confirmation de Mac Donell à la tête du consulat, et ce ne fut qu'après son départ définitif d'Alger ${ }^{11}$ que le gouvernement de Naples décida de confier le poste à un sujet napolitain, Gennaro Magliulo : nommé le 22 février 1824, il avait été chargé précédemment du vice-consulat de Annaba ${ }^{12}$, où il s'était occupé surtout des affaires liés à la pêche du corail, et il dirigea le consulat à Alger (avec des périodes d'intérim assumées pas son fils, Antonio) jusqu'en $1834^{13}$.

9 Sur l'activité des pêcheurs de corail italiens le long de la côte maghrébine, cf. G. Tescione, Italiani alla pesca del corallo ed egemonie marittime nel Mediterraneo, Fiorentini, Napoli 1968 (Ière éd. : Napoli 1940), p. 5-117; J.-L. Miège, Corailleurs italiens en Algérie au XIXe siècle, in Atti della Settimana internazionale di studi mediterranei medievali e moderni, Milano 1980, p. 25-37; T. Filesi, La pesca del corallo nelle acque nordafricane (1734-186o) nelle fonti dell'Archivio di Stato d Napoli, Massimo, Napoli 1985 .

10 Le décret de nomination de De Martino par le gouvernement napolitain est daté du 19 septembre 1816 (cf. I. Caruso, La Reggenza di Algeri: assetto interno e relazioni internazionali nella corrispondenza inedita del consolato del Regno delle Due Sicilie (1816-1827), Edizioni Scientifiche Italiane, Napoli 1990, p. 49).

11 Mac Donell abandonna Alger le 31 janvier 1824, accusé par le gouvernement de la ville de favoriser la rébellion de la Kabylie et d'autres actions contraires à ses intérêts. Après le blocus de la ville par l'escadre commandés par l'amiral Harry Neal, le gouvernement anglais nomma un nouveau consul (William Danfort), à qui le dey prohiba d'assumer la charge consulaire d'autres pays que le sien (cf. C. Piazza, Il Granducato di Toscana e la Reggenza di Algeri (1814-1830), in R.H. Rainero, op. cit., p. 187-188).

12 Magliulo fut en poste à Annaba de 1818 à 1820. (cf. L. Siano, Un diplomatico napoletano alla corte del Dey : a proposito della corrispondenza da Algeri di Gennaro Magliulo, in «Africa» [Rome], XLII, 4/1987, p. 643-649). Parmi les vice-consuls napolitains à Annaba dans les années suivantes, citons l'anglais E. Fowls, nommé en septembre 1833 et un Bensamon (qui était aussi le consul de Sardeigne) à partir de 1846. La Calle aussi hébergea plus tard un consulat napolitain, confié à A. Gebhardt de 1839 à 1854 (cf. A. Bozzo, op. cit., p. 366).

13 Pour l'histoire du consulat pendant cette période, cf. J. Mazzoleni, Il consolato del Regno delle Due Sicilie in Algeri, in « Rassegna Storica Napoletana », II, 4/1934, p. 312-322 ; L. Mazzacca, Le relazioni diplomatiche fra il Regno delle Due Sicilie ed Algeri (1814-1823), Istituto della Stampa, Napoli 1959. Parmi les autres fonctionnai- 
À partir des premières années de la période coloniale un consulat napolitain avait existé à Oran, aussi, dont la date précise d'établissement ne nous est pas connue : jusqu'en 1834 ce poste était dirigé par un vice-consul, Carlo Becciani, et par la suite par Adriano Betti ${ }^{14}$. Au cours de la même année 1834 un nouveau consul fut désigné à Alger, Antonio Girardi ${ }^{15}$, qui y resta pendant quatre ans; au mois de septembre 1838, Giovanni D'Errico prit sa place, et dirigea le consulat jusqu'en 1849 .

3. Les documents napolitains concernant l'émir Abd el-Kader appartiennent à la période 1834-1847 : il s'agit d'une partie des rapports périodiques envoyés au ministère des Affaires étrangères ${ }^{16}$ et qui sont signés, pour la plupart, par les titulaires du consulat d'Alger durant cette période (Girardi et D'Errico) eux-mêmes. On rencontre parfois des rapports des viceconsuls de Bône et d'Oran, mais dans la presque totalité des cas les informations étaient envoyées à Naples à partir du consulat d'Alger. En effet, la correspondance provenant des postes de Bône et d'Oran était normalement dirigée à Alger par le biais des liaisons maritimes régulières qui avaient été établies entre ces villes dès le début de la période coloniale : à Alger, le consul décidait, selon l'importance du cas, d'envoyer une copie de la correspondance reçue par les autres postes, ou bien de la résumer, dans le courrier périodiquement envoyé à Naples.

4. Ce fut Carlo Becciani qui d'Oran envoya en 1834 à la cour de Naples un long rapport dans lequel, pour la première fois, l'émir Abd el-Kader est cité : Becciani, qui le nommait, curieusement, Berkader, en parlait à propos de la

res du consulat de Naples qui nous sont connus par les documents, citons le viceconsul Dionisio De Vecchi (jusqu'à 1841) et Alexander Wendling, qui prit sa place par la suite.

14 Parmi les vice-consuls napolitains à Oran, un nommé Sgitovich (de 1834 à 1836) et Louis Vallin (cf. A. Bozzo, op. cit., p. 154).

15 Qui, avec ses propres mots, fut « le premier consul reconnu légalement pas le gouvernement français » (ibi, p. 122).

${ }^{16}$ La correspondance est envoyé au ministre des Affaires étrangere en personne, c'est-à-dire à «Son Excellence Antonio Statella, Prince de Cassaro, Secrétaire d'État, Ministre des Affaires Étrangères », jusqu'au mois de mars 1841, et par la suite à son successeur, «Son Excellence Fulco Ruffo di Calabria, Prince de Scilla, Duc de Sainte Christine, Conseiller, Ministre d'État, Chargé du portefeuille des Affaires Étrangères ». 
signature du traité Desmichels, intervenue quelque jours auparavant ${ }^{17}$ et qui avait eu un large écho dans les territoires occupés par la France. Par ce traité, Abd el-Kader se voyait reconnaître

le titre de commandeur des croyants, consacrant sa double autorité spirituelle et temporelle, qui ne pouvait le céder à aucune autre [... et le droit de désigner] des consuls (en français, des représentants), ce qui semblait confirmer sa souveraineté. La convention stipulait la fin des hostilités, le respect de la religion et des usages musulmans, la libération des prisonniers français, la liberté de commerce [...] et l'obligation d'un sauf-conduit visé par les consuls de Desmichels et d'Abd el-Kader pour les chrétiens désireux de circuler sans danger ${ }^{18}$.

Voici la traduction de la lettre de Becciani :

L'événement extraordinaire, et auquel personne ne s'attendait, constitué par la paix signée entre les Français et les Bédouins de l'intérieur, des gens superstitieuses et ennemies absolues des Chrétiens, m'encourage à en faire partie à Votre Excellence [...]. Un certain Moïse Busnach, originaire et sujet de Toscane, qui déjà depuis quelque temps fréquente le pays et connaît les moeurs des Arabes, décoré de la Légion d'Honneur et ami de Monsieur le Maréchal Clausell [Clauzel], a été le promoteur et le moteur principal de cette paix. Après avoir écrit plusieurs lettres, ayant reçu une réponse favorable par le Bey Berkader, chef suprême et commandant puissant des Arabes, le susdit Monsieur Busnach accompagné par un commandant français et par Monsieur Amar [Mardoukai] chef de la nation israélite d'Oran, partit le 27 février passé avec deux arabes d'escorte vers le village d'Abrahah, où ils étaient attendus par le susdit chef Berkader. Ils arrivèrent au susdit village après avoir parcouru vingt lieu d'un territoire qui a été plusieurs fois arrosé du sang Français et Bédouin, chemin dangereux pour son insécurité, et qu'il y a vingt jours aurait été le théâtre de l'assassinat de quiconque eut voulu le parcourir. En présence de Berkader, les

17 La convention, qui fut appelée plus tard le traité Desmichels, avait été signé le 26 février et ses « deux textes, français et arabe, accusaient de profondes différences » (Ch.-A. Julien, Histoire de l'Algérie contemporaine, t. I : La conquête et le début de la colonisation (1827-1871), p. 104).

18 Ibidem. 
ambassadeurs furent accueillis avec tous les honneurs militaires usuels pour la Nation Arabe : une musique barbare et des salves de mousquets les saluèrent : ils trouvèrent ici cinq prisonniers Français que Berkader avait fait venir pour les rendre à leurs Drapeaux, quel que fût le résultat des tractations, et cela afin de prouver l'estime qu'il dit avoir du Général en Chef Monsieur le Baron Desmichels.

Après un séjour de quatre jours avec Berkader, comblés d'honneurs et d'égards, et lorsqu'ils se préparaient à retourner à Oran, Berkader les pria de bien vouloir l'accompagner jusqu'au village de Segh [Sigh], cinq lieus plus loin. Pendant ce court voyage la suite de Berkader, qui comptait alors 200 hommes, augmenta jusqu'à six mille chevaliers. Cette escorte nombreuse était accompagnée par plusieurs orchestres militaires qui avec leurs instruments barbares alternaient des marches avec des décharges fréquentes de mousquets en signe de joie. Arrivés à Segh, les deux groupes se séparèrent, en se dirigeant du côté d'Oran avec Milùd Ben Arasch, Agha de Berkader qu'il a déjà envoyé comme plénipotentiaire en compagnie d'autres chefs de tribus et avec une escorte d'Arabes. Le chemin de Segh aux environs d'Oran était encombré par les chevaliers Arabes qui saluaient en continuation les voyageurs avec des décharges de mousquets.

La soirée du quatre [du mois] courant les Députés Français et Arabes accompagnés par les prisonniers entrèrent dans la ville au milieu des acclamations de toute la population de la garnison sortie à leur rencontre. La soirée qui fit suite à la première réunion (c'est-à-dire le six courant) un grand bal fut organisé dans la résidence du général en chef à la présence de toutes les autorités, et des Bédouins qui furent très surpris de voir des femmes si peu couvertes et si publiquement courtisées.

Le bal eut en très grand succès, et se prolongea très tard dans la nuit. Le sept une grande parade fut organisée et toutes les troupes Françaises réunies firent une petite guerre avec la satisfaction et le plaisir des susdits Bédouins. Tout le monde a été surpris par cette paix, puisque l'on connaît la haine des kabyles de ces Arabes et leur superstition religieuse. Quel que soit le but des Arabes, on pourra compter et se fier à la Foi de Berkader dont les ancêtres ont plusieurs fois régné sur ces provinces, et pour l'instant dans ce pays [la convention] acquiert un fort intérêt pour le commerce courant, puisque l'un des principales conditions entre autres concerne la liberté du commerce pour les deux nations. Berkader, dont la puissance s'étend bien au-delà d'Alger à 
partir d'Oran, et qui peut réunir en deux jours sous ses ordres cinquante mille chevaliers, a garanti [l'arrêt] des assassinats et la sécurité des chrétiens et de leur liberté. Les massacres et les tueries continuelles pourront ainsi terminer : ainsi le pays ne manquera plus de vivres, étant restés plusieurs fois dans le passé sans viande, oeufs et poulets. Cela offrira des avantages utiles pour le commerce, en ouvrant ainsi les communications avec l'Empire du Maroc aussi $[\ldots]^{19}$.

Le texte que voici nous donne un exemple de la typologie d'informations et de renseignements que l'on peut trouver dans les documents napolitains concernant les événements de la période qui vit la lutte entre les troupes d'occupation françaises et les tribus du territoire algérien ralliées sous la bannière de l'émir Abd el-Kader.

Ces documents ne révèlent que très rarement les sources de leurs informations, mais, puisque les agents consulaires napolitains n'avaient pas d'accès direct au champ de l'émir, il paraît évident que leurs informations étaient pour la plupart tirées des sources françaises, soit à travers les communications officielles, soit à travers les colloques qu'ils avaient avec les fonctionnaires du gouvernement colonial, soit par les publications et la presse périodique. En de rares cas, les informations transmises à Naples avaient été recueillies à travers un contact direct avec les italiens résidant dans le territoire : en particulier, avec le capitaine Raffaele Poerio, sujet du royaume de Sardaigne, qui est cité plusieurs fois dans deux lettres envoyées à Naples ${ }^{20}$. En particulier - il est important de le souligner, parce que les renseignements sur les événements militaires sont assez fréquents dans cette correspondance - il ne faut pas oublier qu'une remarquable quantité d'italiens (beaucoup d'entre eux étaient des déserteurs qui avaient fui le territoire de la péninsule pour des raisons politiques, à cause de la répression contre les mouvements insurrectionnels et les organisations politiques secrètes qui se battaient pour l'indépendance italienne pendant

19 ASN, AE, 2382: lettre du 10.3.1834 envoyée d'Oran par le vice-consul Carlo Becciani au Prince du Cassaro, ministre des Affaires étrangères. Pour le texte original en italien, cf. Appendice.

20 Poerio est sûrement le « capitaine sarde de la légion étrangère », qui est cité comme source des renseignements sur la bataille de la Macta dans une lettre du 11 septembre 1835. Dans la même lettre, on annonce la promotion probable de Poerio pour sa bravoure au cours de la même bataille. 
le Risorgimento ${ }^{21}$ ) s'était engagée en Algérie dans l'armée française : à un tel point qu'un régiment italien de la Légion Étrangère avait été formé, qui batailla à plusieurs reprises contre les troupes de l'émir ${ }^{22}$.

À partir de ce premier document concernant Abd el-Kader, dans les années successives les lettres du consulat d'Alger renseignant le ministère des Affaires étrangères de Naples sur les événements algériens liés à l'affirmation de la puissance de l'émir et à ses combats contre les troupes françaises vont se multipliant.

Après le traité Desmichels, Abd el-Kader avait rallié plusieurs tribus à sa cause et réorganisé sa force militaire: dans un document daté du mois de juin 1835 on peut lire qu'il

dispose actuellement de 12.000 hommes fournis par les différentes tribus [... et qu'il dispose d'un apparat militaire] formidable. Il n'y a pas de guerre contre les Français ni d'hostilité, mais ils s'observent de loin ${ }^{23}$.

D'après le consul Girardi, cette situation ne pouvait pas durer longtemps, puisque l'émir (dont on disait qu'il avait l'ambition de s'emparer de Constantine) ne cessait d'augmenter ses forces par le recrutement et l'imposition de contributions aux tribus, ce que les Français devaient tolérer parce qu'ils n'étaient pas à mesure de l'en empêcher. Et, en effet, moins d'un mois plus tard une lettre de Girardi décrit les opérations qui ont donné lieu à la bataille de la Macta, où Abd el-Kader a écrasé une colonne française, et ses conséquences. Les renseignements contenus dans cette lettre proviennent du capitaine Raffaele Poerio, dont nous avons déjà parlé, qui les a transmis au vice-consul napolitain d'Oran:

21 Cf. E. Michel, Esuli italiani in Algeria, Cappelli, Bologna 1935, passim.

22 Par une ordonnance royale de 1831 une légion constitué d'étrangers et qui ne pouvait pas être utilisée qu'à l'extérieur du territoire métropolitain français fut instituée, dans laquelle pouvaient s'engager des volontaires admis par le commandant en dehors des pratiques administratives courantes dans l'armée. En 1833 le bataillon italien se trouvait à Alger. Plusieurs déserteurs italiens de la légions passèrent par la suite dans l'armée d'Abd el-Kader (cf. Ch.-A. Julien, op. cit., p. 271272).

23 ASN, AE, 2381: lettre du consul Girardi au Prince de Cassaro, ministre des Affaires étrangères, 27.6.1835. 
Trézel, commandant la province, était sorti [du champ de Tlélat, à quelques dizaines de kilomètres au sud d'Oran ${ }^{24}$ ] avec 2.500 hommes pour attaquer Abd-el Kader qui avait levé des contributions et molesté les tribus soumises aux Français. Pour se défendre contre lui, elles avaient reçu des armes et des munitions des Français, et Abd-el Kader aussi en avait reçues précédemment. Ils [les hommes des tribus] s'étaient tous réunis, et le général Trézel avec sa petite armée se trouva confronté avec plus de 20.000 mores commandé par Kader, qui avaient pris un canon, tous les caissons à l'exception d'un, et 120 milles cartouches; ils avaient tué 1.500 Français et le général Trézel s'était réfugié à Arzeo $[\text { Arzew }]^{25}$.

Suite à cette bataille, continuait Girardi, Oran se trouvait dégarnie, et en cas d'attaque on aurait pu la défendre avec beaucoup de difficulté : tous les bateaux disponibles avaient été alors envoyés à Arzew pour ramener les troupes qui y étaient bloquées. Les informations officielles diffusées plus tard par le gouvernement général limitaient les pertes françaises à 600 hommes (dont un colonel et 10 officiers), tandis que l'émir en avait perdus 2.00o. La lettre de Girardi terminait relatant les critiques qui étaient faites au gouverneur pour avoir fournis les armes aux algériens, tandis que Trézel était accusé d'avoir mal dirigé les opérations; Abd el-Kader par contre avait montré de grandes qualités de commandement, et ses troupes une rapidité remarquable de déplacement et d'action.

L'émir avait par la suite écrit au gouverneur général Drouet d'Erlon affirmant que Trézel était le responsable des ces événements : quelques temps plus tard celui-ci avait été remplacé par d'Arlanges ${ }^{26}$.

Analysant la situation qui s'était créée suite à la bataille de la Macta, Girardi soulignait l'insécurité du pays, en particulier dans les alentour d'Alger (où les moissons des colons français avaient été incendiées), ainsi que «l'indifférence des Français aux dangers auxquels ils s'exposent» et «la protection que l'on accorde à Abd-el Kader » ${ }^{27}$ : il en déduisait, en observant que les Chambres parisiennes lors des derniers débats avaient

24 Cf. Ch.-A. Julien, op. cit., p. 126. D'après Julien, la colonne était composé de 2.00o hommes et avait perdu à la Macta le quart de ses effectifs : la bataille avait eu lieu le 28 juin.

25 ASN, AE, 2381 : lettre du 11.7.1835.

26 Que Girardi cite comme Darlange dans sa lettre du 18.7.1835.

27 ASN, AE, 2381: lettre du 18.7.1835. 
montré une très forte hostilité quant au projet de conserver le territoire conquis, que peut-être l'émir était destiné à devenir le souverain du pays. Cela aurait pu constituer la solution du problème, puisque d'après les opinions courantes les avis étaient partagés sur le sort de l'Algérie après l'abandon par la France.... Et le consul de conclure:

Peut-être cette opinion n'est que l'un de mes rêves ${ }^{28}$.

On peut affirmer, à partir de ce dernier document, que dans le consulat napolitain prédominait une attitude favorable à l'affirmation de l'émir, ou en tout cas défavorable à la continuation de la présence française en Algérie. Il ne faut pas oublier, pour comprendre cela, que déjà avant l'expédition de 1830 les intérêts français et napolitains dans les territoires nord-africains avaient été en opposition, par exemple dans le secteur de la pêche du corail, ni que la politiques étrangère du royaume des Deux Siciles était depuis longtemps dictée par l'Angleterre (à laquelle la dynastie napolitaine était très fortement débitrice, surtout depuis les guerres napoléoniennes), dont l'hostilité contre la présence française au Maghreb était encore très forte.

Dans la deuxième moitié de 1835 et dans la première de l'année suivante les rapports provenant d'Oran contiennent des références, de valeur différente, aux opérations militaires de l'émir: entre les mois de juillet et septembre, après que les pertes subies dans les opérations contre la colonne de Trézel ont été comblées, on relate les escarmouches dans les alentours de la ville et les attaques contre les tribus alliées des Français. Abd el-Kader compte alors une armée de 25.000 hommes, et ses ennemis ne peuvent pas organiser une riposte faute de forces suffisantes ${ }^{29}$. Mais déjà au mois de septembre la France prépare un corps expéditionnaire de 11.000 hommes pour contre-attaquer : on dit qu'il sera commandé par le duc d'Orléans, qui en effet débarque à Oran le 21 novembre.

Les troupes françaises entrent en action à la fin du même mois : il s'agit de quatre division de 11.158 hommes au total, avec 2.000 chevaux, 700 chameaux chargés de vivres pour 22 jours et 18 canons Chaque soldat est fourni de 100 cartouches, et la réserve en a 800 milles $^{30}$. Après avoir outrepassé le wâdî Sigh, la colonne bat de son artillerie les arabes qui sont venus à sa

\footnotetext{
28 Ibidem.

29 ASN, AE, 2381 : lettres des $25 \cdot 7$ et 12.9.1835.

30 ASN, AE, 2381 : lettres des 29.10 et 1.12.1835.
} 
rencontre : c'est un véritable massacre. Abd el-Kader se retire 25 milles au sud de Mascara, qui est occupée par les français non sans rencontrer une résistance au cours de laquelle le général Oudinot est blessé. L’avancée de l'ennemi a contraint beaucoup de tribus à la soumission, tandis que même l'un des aghas de l'émir s'est rendu ${ }^{31}$.

Le 12 janvier 1836 c'est le tour de Tlemcen : 8.000 hommes sous le commandement du maréchal Clauzel, accompagnés par 1.000 «turcs » (c'està-dire kouloughlis) aux ordres de Mustafa ben Ismail, entrent dans la ville presque sans rencontrer d'opposition ${ }^{32}$. Dans le rapport sur la prise de Tlemcen du vice-consul d'Oran, qui accompagne la lettre envoyée à Naples par Girardi, on voit entrer en scène pour la première fois les combattants marocains qui sont venus à l'aide d'Abd el-Kader. Pendant l'occupation de la ville - on raconte - des espions de l'émir ont été capturés qui ont permis à Clauzel de savoir que

7.00o marocains sont venus à la défense contre l'ennemi ; ceci amènera peut-être à des explications entre la France et le Maroc; bien que jusqu'à maintenant on ne peut pas savoir si les troupes de ce dernier étaient sous le commandement d'un chef marocain ${ }^{33}$.

Dans les mois successifs les troupes coloniales avaient occupé Blida, Médéa et Miliana, qui toutefois avaient été conservées seulement pour une courte période. Le territoire tout autour était toujours contrôlé par l'émir, qui avait été vu à plusieurs reprises non loin de Tlemcen accompagné de ses hommes et qui n'avait pas tardé à riposter aux attaques. Le 25 avril il avait fait subir un grave revers à une colonne française de 2.000 hommes qui sous la conduite de d'Arlanges se trouvait non loin de l'embouchure de la Tafna: 600 soldats avaient été tués ou blessés, dont 37 officiers, tandis que le reste de la colonne était bloquée et ne pouvait être ravitaillée que par mer. Dans le document envoyé à Naples relatant cette affaire on affirme que lors de cette bataille beaucoup de marocains se trouvaient dans les rangs d'Abd el-Kader, que ses troupes ont capturé deux canons et qu'elles se sont

31 ASN, AE, 2381 : lettres des 7.12 et 14.12.1835 et du 9.1.1836.

32 ASN, AE, 2381: lettre du 21.2.1836. D'après le consul Girardi, on a compté 80 blessés parmi les troupe française au cours de cette expédition.

33 Ibidem. La présence de beaucoup de combattants marocains dans l'armée de l'émir est cité aussi dans une autre lettre du 21.5.1836 (ASN, AE, 7051 ; cf. A. Bozzo, op. cit., p. 256). 
battues avec une valeur extrême à la baionnette: cela signifie qu'elles possédaient désormais un entraînement moderne qui leur permettait, en quelques situations, de se confronter à égalité avec l'armée française ${ }^{34}$.

Tandis que les troupes bloquées à la Tafna attendaient les 5.00o hommes envoyés de France sous le commandement de Bugeaud pour leur libération, à Alger dix parmi les plus influents notables arabes de la ville, dont « le fameux Bouderba et Mustapha Pacha», avaient été arrêtés et envoyés à Bône sous escorte: on affirmait, selon les informations du consul napolitains, qu'ils avaient ourdi une conspiration en faveur d'Abd el-Kader ${ }^{35}$.

Bugeaud avait réussi à débloquer la situation du camp fortifié de la Tafna et à battre Abd el-Kader dans une bataille que l'on disait désastreuse pour l'émir : d'après la relation de cette bataille envoyée à Naples, il avait compté plus de 800 morts et 117 prisonniers parmi ses hommes, tandis que Bugeaud n'avait perdu que 12 hommes morts, 20 blessés et 50 chevaux. On disait encore qu'Abd el-Kader lui-même avait reçu deux blessures mortelles dans les combats, mais ce bruit avaient été démenti quelques jours plus tard ${ }^{36}$.

À l'occasion de l'arrivée des troupes de Bugeaud, le gouvernement français avait commencé les manoeuvres d'intimidation contre le sultan du Maroc, afin d'empêcher que les marocains s'engagent encore plus du côté de l'émir: les bateaux de guerre qui avaient transporté les soldats étaient allés faire une démonstration navale en face de $\operatorname{Tanger}^{37}$. On supposait, du côté français, que l'intervention marocaine faisait partie d'un complot ourdi par les États-Unis, le sultan du Maroc et Abd el-Kader.

Le bruit d'une intervention américaine dans le conflit s'était répandu à Oran lorsque le capitaine d'un bateau arrivé de Gibraltar fin juin 1836 avait affirmé avoir croisé le long de sa route une escadre au pavillon des ÉtatsUnis: afin de contrecarrer une éventuelle manoeuvre américaine, des

34 ASN, AE, 2381 : lettres des 29.3, 10.4 et 21.5.1836. Toutefois, la faiblesse de l'armement de l'émir rendait impossible la confrontation avec l'armée française lorsque l'artillerie était en jeu.

35 ASN, AE, 2381: lettre du 4.6.1836. D'après une autre hypothèse, leur arrestation aurait été la conséquence des lettre de dénonciation contre le gouverneur qu'ils avaient envoyé à Paris.

36 ASN, AE, 2381 : lettres des 23.7 et 30.7.1836. La bataille de la Sikkak, dont on parle ici, avait eu lieu le 6 juillet : cet exploit avait permis à Bugeaud de ravitailler non seulement le camp de la Tafna, mais aussi la ville de Tlemcen, qui entre temps avait été bloquée par les troupes de l'émir.

37 ASN, AE, 2381: lettre du 14.6.1836. 
bateaux de guerre français croisaient depuis lors dans les eaux de Mers el-Kebir. Le consul napolitain relate ainsi le 'complot américain' :

On suppose que l'escadre [des États Unis] avait l'intention de s'emparer des îles Schiaffarines, qui ne sont pas habitées, [et qui se situent] entre l'état du Maroc et Tlemsen [Tlemcen], et qui ont été achetées il y a quelque temps par les États-Unis à l'empereur du Maroc. Je peux affirmer que les autorités françaises suivent les traces d'un complot entre les Américain, le Maroc et Abd el-Kader, dont le but ne paraît pas pouvoir être que d'obtenir le port dans la Méditerranée qu'ils désirent si fort. Faute de mieux, Mars el Kebir pourrait être leur objectif, mais, même s'ils arrivaient à s'en emparer, il suffirait une journée pour les en chasser, puisque ce port est totalement encerclé par les collines et par conséquent il peut être facilement foudroyé [par l'artillerie] ${ }^{38}$.

D'un 'complot américain', ou en tous les cas d'une manoeuvre secrète entre les États-Unis et les ennemis de la France en Algérie (dont Abd el-Kader, en particulier), on parla à nouveau quelques temps plus tard, après la signature de la convention de la Tafna ${ }^{39}$. Cette convention prévoyait, entre autres, un échange de représentants entre le gouvernement colonial et l'émir, et déjà au mois d'août les français avaient choisi la commandant

38 ASN, AE, 2381 : lettre du 2.7.1836 (sur le même sujet, ASN, AE, $705^{1}$ : lettre du 16.7.1836). Les tensions entre la France et les États Unis étaient très forte à cette époque : dans une lettre du consulat d'Alger du 14.11.1835 on donnait pour probable une guerre entre les deux pays (ASN, AE, 7051 : cf. A. Bozzo, op. cit., p. 254). Les « îles Schiaffarines » (Chaffarines ou Zaffarines en français, Chafarinas en espagnol, probablement du nom arabe Zafran, ou bien du nom de la tribu berbère des Yaffarines) sont trois îlots situés en face de la côte du Maroc, non loin de l'embouchure de la Muluya, et à 45 kilomètres à l'est de Melilla. Elles furent occupées par l'Espagne (qui y établit un pénitencier et qui encore aujourd'hui les occupe) en 1848.

39 À la convention de la Tafna, signée le 30 mai 1837 par l'émir et Bugeaud, la correspondance du consulat napolitain dédie peu d'attention, en estimant « qu'elle ne durera que l'espace de temps qu'Abd-el Kader jugera convenable pour lui, c'està-dire jusqu'à ce que les Arabes auront assuré la sécurité de leurs moissons de blé et d'orge » (ASN, AE, 2382 : lettre du 24.6.1837). Le texte de la convention est envoyé à Naples (avec une copie du «Moniteur Algérien » du 28.7.1937 qui le reproduit) par une lettre du 5.8.1837, dans laquelle on affirme qu'elle « ne satisfait personne ». Par contre, le vice-consul d'Oran s'empresse de souligner que, suite à la signature de la convention, la ville se voit enfin ravitaillée en abondance de vivres de toute sorte et que les prix ont baissé de façon remarquable : jusqu'à quatre fois pour la viande, et en proportion pour les autres victuailles (lettre du 26.8.1837). 
Menonville, qui avait été accepté par l'émir et qui s'était rendu à Mascara ${ }^{40}$. Quelque temps plus tard l'émir avait désigné comme oukil à Alger un italien, Carlo Garavini : Garavini, que le consul napolitain présente dans l'une de ses lettres comme « un commerçant [originaire] de Modena ${ }^{41}$, était le consul des États-Unis à Alger.

Cette désignation avait rencontré l'opposition du gouvernement français: l'intendant civil Bresson avait fait remarquer au gouverneur que Garavini aurait pu d'un côté agir comme un espion pour le compte de l'émir, et d'un autre côté favoriser les États-Unis dans leur but d'acquérir une base navale sur la côte de l'Algérie ${ }^{42}$. On avait envisagé la possibilité de l'expulser, mais cela aurait créé des problèmes de caractères diplomatiques avec les américains.

Tandis que les officiels du gouvernement colonial discutaient de la stratégie à mettre en place pour conjurer les supposés dangers représentés par la désignation de Garavini, celui-ci avait reçu les instructions de l'émir et pendant une audience avec le gouverneur général, le maréchal Valée, avait présenté de sérieuses doléances sur le manque de respect de certaines clauses de la convention, en demandant que les français résolvent au plus tôt les problèmes posés par leur attitude négative ${ }^{43}$. Immédiatement après cet épisode, le gouvernement général avait communiqué qu'il n'acceptait pas la nomination de Garavini, prétextant, entre autres, que, comme ils avaient

40 La présence de Menonville à Mascara s'était terminée par une tragédie: accusé d'avoir fait empoisonner, au lieu de le soigner, l'un des enfants d'Abd elKader, il avait tué son interprète et s'était suicidé. Le consul napolitain raconte brièvement cet épisode dans une lettre du 7.11.1837.

41 ASN, AE, 2382 : lettre du 23.10.1837. Le personnage est cité comme « un certain Garavini, consul des États-Unis » dans S. Aouli, R. Redjala, Ph. Zoummeroff, Abd el-Kader, Fayard, 1994, p. 217.

42 Ibi, p. 217. La crainte française de l'intervention d'une autre puissance navale le long des côtes algériennes était manifeste aussi dans la convention de la Tafna, où un pouvait lire, à l'art. 13 du texte français, que «l'Émir s'engage à ne concéder aucun point sur le littoral à une puissance quelconque sans l'autorisation de la France ». Sur les intérêts et sur la politique des États Unis dans la Méditerranée des débuts du XIX ${ }^{\mathrm{e}}$ siècle $\mathrm{cf}$. P. Soave, La rivoluzione americana nel Mediterraneo : prove di politica di potenza e declino delle Reggenze barbaresche (1795-1816), Giuffré, Milano 2004, qui toutefois ne traite pas de la période qui nous intéresse.

43 «M. Garavini obtint une audience par le Général Valée, lui présentant des réclamations plutôt sérieuses de la part d'Abd-el Kader, qui avait ordonné d'une façon ou d'une autre à son consul de demander que l'on redresse les torts dont il se plaint, et qui, comme l'on dit, avait mis le marché à la main [en français dans le texte] aux Français » (ASN, AE, 2382: lettre du 9.12.1837). 
envoyé un français à Abd el-Kader, « ils voulaient avoir [de lui] pour consul un Arabe pur $»^{44}$.

Par une lettre indignée, l'émir avait répondu à Valée qu'il n'avait aucun droit d'intervenir sur son choix ${ }^{45}$, tandis que dans une autre lettre à Garavini il affirmait qu'il n'avait aucune intention d'envoyer un consul arabe à Alger « parce que ses Arabes, trop simples, auraient été bientôt les victimes de l'astuce française $»^{46}$.

Suite à la réponse piquée de l'émir, le gouverneur général avait laissé tomber l'affaire et choisi une autre stratégie pour se débarrasser de Garavini. Celui-ci avait été autorisé à se rendre à Mascara et dans les territoires hors de la juridiction française ${ }^{47}$, ainsi qu'à rencontrer Valée pour lui transmettre les messages de l'émir, toutefois les autorités françaises avaient maintenu la vigilance. Les suspicions sur les dangers que l'oukil italien pouvait faire courir aux intérêts français avaient été confirmées lorsqu'on avait arrêté à Alger (et par la suite expulsé) un fabriquant d'affûts de canon débarqué de Livourne qui lors de son interrogatoire avait déclaré avoir été engagé par Garavini pour se mettre au service d'Abd el-Kader ${ }^{48}$.

Le bruit avait alors couru dans les chancelleries consulaires d'Alger que Garavini aurait été expulsé immédiatement, mais il n'en avait rien étée ${ }^{49}$ : par contre, environ un mois plus tard, pour des raisons que le consul napolitain définissait « un mystère ${ }^{50}$, le gouvernement français avait annulé, après en avoir obtenu l'accord par l'ambassade des États-Unis à Paris, l'exequatur qui l'autorisait à remplir les fonctions consulaires pour ce pays, et

44 Ibidem.

45 Cf. G. Yver, Documents relatifs au traité de la Tafna (1837), Carbonnel, Alger 1924, p. 326.

46 ASN, AE, 2382 : lettre du 23.12.1837.

47 Garavini avait rencontré l'émir à Médéa début 1838 (cf. A. Bozzo, op. cit., p. 262-263).

48 ASN, AE, 2382: lettre du 27.1.1838. Un autre épisode semblable, ou bien le même, mais raconté différemment, se trouve dans la biographie de l'émir par Ch.-H. Churchill (The Life of Abd el-Kader, ex sultan of the Arabs, Chapman Hall, London 1876), où l'on relate la fermeture de la boutique et l'expulsion d'un fabriquant de roues, dont l'armée d'Abd el-Kader se servait pour les affûts de ses canons. Dans le même livre (p. 163 et suivantes) on trouve le texte des protestations de l'émir à Valée, qui lui avait communiqué que Garavini était persona non grata pour le gouvernement français, et plusieurs autre références à l'oukil italien.

49 Ibidem.

50 ASN, AE, 2382 : lettre du 24.2.1838. 
quelques jours plus tard il avait été obligé d'enlever du toit de sa maison un petit drapeau américain ${ }^{51}$.

Les documents napolitains ne nous permettent pas de savoir s'il continua à exercer la charge d'oukil kadirien encore pour quelque temps ou bien s'il fut expulsé immédiatement après d'Alger ${ }^{52}$ : on peut toutefois émettre l'hypothèse, à partir de ces documents, que toute somme faite la 'motivation américaine' n'avait pas compté, dans l'opposition à Garavini de la part des autorités françaises, autant que son activité trop favorable à l'émir, en particulier dans le secteur de l'armement.

En effet, bien que la convention de la Tafna prévoyait une augmentation des moyens militaires à la disposition de l'émir, le gouvernement colonial n'était pas du tout disposé à l'admettre, et non seulement dans le cas de l'artillerie, qui constituait la raison principale de sa supériorité militaire. D'où, au-delà de l'épisode du fabriquant livournais d'affûts de canon, les réclamations continuelles de l'émir pour que les clauses de la convention relatives à l'armement fussent respectées, comme le démontre, au mois de février 1838 , sa demande réitérée pour la fourniture de 12 tonnes de poudre pour les armes à feu que le gouvernement français s'était engagé à lui fournir d'après la convention, mais dont l'envoi avait été suspendu sine $\mathrm{di}^{53}$.

Dans cette situation, tous les moyens étaient bons pour l'émir afin de se doter des armes nécessaires pour la consolidation et l'extension de son pouvoir : d'où l'augmentation de la contrebande le long de la côte occidentale de l'Algérie, dont le gouvernement général accusait les Anglais, les Toscans et les Sardes ${ }^{54}$. D'un autre côté, Abd el-Kader avait besoin, pour mieux organiser le nouvel état qu'il avait fondé, de ne pas interrompre la période de paix qui avait fait suite à la convention : d'où ses manifestations d'amitié pour la France, telle l'envoi de cadeaux au roi Louis-Philippe du mois de février 1838. À cette occasion - on lit dans une lettre envoyée à Naples — son khalifa Miloud Ben Arach était arrivé à Alger, avant de partir pour Paris, avec quatre autruches, 80 chevaux, dont 23 de très grande race, plumes d'autruche, tapis et d'autres objets manufacturés arabes ${ }^{55}$. Enfin, avec un travail secret qui devait lui permettre de renforcer son armée, l'émir avait «fait diffuser en sourdine une proclamation, avec laquelle il

51 ASN, AE, 2382 : lettre du 10.3.1838.

52 Comme il semblerait à la lecture de S. Aouli et al., op. cit., p. 218.

53 ASN, AE, 2382 : lettre du 24.2.1838.

54 Ibidem.

55 Ibidem. Cf. aussi Ch.-A. Julien, op. cit., p. 148. 
invite tous les Musulmans à se joindre à lui, avec mille promesses » ${ }^{56}$. Cette propagande avait eu quelques succès à Alger, puisque elle avait produit «en une semaine l'émigration de 300 personnes de la ville ${ }^{57}$.

Tandis que, avec une action aux multiples facettes, l'émir renforçait son pouvoir dans la région occidentale, la France avait subi à l'est une sévère défaite lors de la première expédition contre Constantine (novembre 1836): les documents napolitains provenant de Bône contiennent quelques notes sur les préparatifs, le départ et le désastre de l'expédition, qui avait fait penser à la possibilité qu'Abd el-Kader puisse réaliser une entente avec Hajj Ahmed, le bey qui gouvernait la région de Constantine et qui avait repoussé l'attaque, modifiant ainsi de façon profonde l'équilibre des forces qui se confrontaient en Algérie ${ }^{58}$.

Par la suite le gouvernement français avait décidé une deuxième expédition pour s'emparer de la capitale de l'est, grâce aussi à la trêve dans le secteur occidental assurée par la convention de la Tafna: les premières nouvelles de la préparation de cette expédition étaient parvenues à Naples au courant du mois de septembre 1837, lorsqu'une lettre du consul d'Alger avait communiqué le refus des propositions de paix françaises par Ahmed Bey, « poussé par les tribus »59. D'autres lettres avaient annoncé la prise de Constantine, où Ahmed Bey n'avait laissé que «quelques tapis et [... ses] femmes $»^{60}$, et où la France aurait placé une garnison de 3.000 hommes $^{61}$.

Au cours des premiers mois de 1838 , malgré la mission à Paris de Miloud Ben Arach, les rapports entre l'émir et le gouvernement général (qui demandait une révision des limites des territoires de souveraineté reconnus par le traité de la Tafna) étaient allés empirant, et déjà au cours du mois de mars le consul Girardi avait envoyé une lettre très pessimiste à Naples, dans laquelle il affirmait que les hostilités auraient bientôt repris ${ }^{62}$.

56 ASN, AE, 2382 : lettre du 24.2.1838.

57 Ibidem.

58 ASN, AE, 2381 : lettres des 15.10, 29.10, 7.12.1837.

59 ASN, AE, 2381: lettre du 26.8.1837.

60 ASN, AE, 2382 : lettre du 7.11.1837. Cf. aussi ASN, AE, 7052 : lettre du 29.10.1847, où l'on dit au contraire que le palais du bey a été trouve plein d'argent et d'objets précieux.

61 Plusieurs rapports provenant du vice-consul napolitain à Bône, E. Fowls, traitent de la situation de la région de Constantine avant et après la conquête française (sur les documents concernant Constantine, cf. aussi A. Bozzo, op. cit., p. 126-127 ; 258-262).

62 ASN, AE, 2382 : lettre du 10.3.1838. 
Toutefois, au cours de la partie restante de cette année il n'y eut pas de nouvelles confrontations armées entre les deux parties, tandis que l'émir se battait contre ses adversaires algériens : à la fin du mois d'octobre on le trouve au siège de «El-Media [c'est-à-dire d'Ain Mahdi], à six jours de chemin au-delà des confins de cette ancienne Régence ${ }^{63}$. Ce ne fut qu'à la fin de 1839 que les hostilités furent ouvertes à nouveau, lorsque le gouvernement général crut pouvoir imposer par la force une révision du traité défavorable à Abd el-Kader et qu'il avait refusé d'accepter ${ }^{64}$. Le 18 novembre l'émir annonça la reprise de la guerre, et dans un espace de temps très court les territoires contrôlées par la France se trouvèrent dans un état d'insécurité totale: 400 colons, surtout dans les environs d'Alger, furent tués à l'occasion des attaques ${ }^{65}$.

La riposte française ne tarda pas : au mois de mars une colonne occupa Cherchell tout en poursuivant sa marche vers Tenès, tandis que d'autres colonnes mobiles, au cours des mois suivants, se confrontaient avec les troupes kadiriennes et les battaient ${ }^{66}$. La nouvelle guerre avait eu des retentissements dans les pays voisins, et le consul napolitain faisait remarquer la sympathie des tunisiens et des marocains pour la cause de l'émir ${ }^{67}$, qui avait proclamé la guerre sainte ${ }^{68}$.

L'arrivée de Bugeaud à Alger comme gouverneur général, annoncée à Naples par une lettre du 23 février $1841^{69}$, avait fait penser tout de suite que d'importants changements se préparaient : le nouveau gouverneur avait en effet fait diffuser, tout de suite après son arrivée, une proclamation dans

63 ASN, AE, 2383 : lettre du 20.10.1838.

64 Plusieurs lettres sur les tentatives françaises d'imposer une révision des clauses territoriales du traité et sur leurs provocations militaires (telles que l'expédition aux Portes de Fer) : ASN, AE, $705^{2}$ : lettres des 16.3, 2.11, 6.11, 24.11.1839.

65 ASN, AE, 2382 : lettre du 29.12.1839. Cf. aussi Ch.-A. Julien, op. cit., p. 151: «Aussitôt [après le 18 novembre] réguliers de son armée, Hadjoutes et Kabyles de son khalifa Ben Salem se ruèrent sur la Mitidja où ils ruinèrent, en peu de jours, les efforts de colonisation de plusieurs années ».

66 Par exemple, non loin du camp de Kara Mustapha, où le khalifa Ben Salem fut battu (ASN, AE, 2384: lettre du 22.9.1840). Pour l'occupation de Cherchell, cf. ibi, lettres des 28.2 et 21.31839 .

67 ASN, AE, 2384 : lettre du 22.9.1840.

68 ASN, AE, 7052 : lettre du 4.1.1840.

69 ASN. AE, 2384. Bugeaud avait été nommé le 29 décembre; il était arrivé à Alger le 22 février. 
laquelle on annonçait la décision du gouvernement français de développer la colonisation ${ }^{70}$.

Bugeaud avait rencontré quelques jours plus tard le consul D'Errico et lui avait exposé son plan de «colonisation armée ${ }^{71}$. Le plan prévoyait la construction de nouveaux villages dans les régions les plus adaptées au développement agricole et lors de l'entrevue le gouverneur avait manifesté son désir que le royaume de Naples participe à la réalisation de ce projet, par exemple par l'envoi d'un corps de troupes auxiliaires. Il aurait pu compter, affirmait Bugeaud, jusqu'à 6.000 unités: en échange il disait que la France était disposée à offrir des possessions le long de la côte, et avait indiqué à ce propos les villes de Bougie et de Gigelly [Jijel]. Il avait prié D'Errico de communiquer cette proposition à la cour de Naples, ce que le consul avait fait en y ajoutant ses considérations personnelles : il déconseillait fermement d'accepter l'offre de Bugeaud, en considérant qu'elle avait été uniquement dictée par le besoin d'augmenter la disponibilité des forces militaires sous ses ordres, et qu'en tout cas il n'aurait jamais maintenu ses promesses ${ }^{72}$. Quelque mois plus tard le gouvernement napolitain avait en effet répondu négativement à l'offre de Bugeaud, en envoyant au consul des instructions pour qu'il refuse tout accord proposé par le gouvernement colonial pour la participation de l'armée napolitaine au projet de colonisation $^{73}$.

70 ASN, AE, 7053, lettre s.d. [février 1841 ?]. Cf. A. Bozzo, op. cit., p. 270.

71 ASN, AE, 2384: lettre du 2.3.1841. Le plan, qui prévoyait la réalisation d'un fossé défendu par 62 blockhaus autour du territoire à coloniser, à partir de Maison Carrée, est exposé en détail dans une lettre successive (ASN, AE, 2384: lettre du 26.7.1841). On cite un projet destiné à offrir une plus grande sécurité aux colons, avec la construction « d'une grande muraille autour des villes les plus importantes », dans une lettre du 7.6.1840 (cf. A. Bozzo, op. cit., p.138).

72 Ibidem. Ce n'était pas la première fois qu'un gouverneur français proposait au royaume napolitain de participer à la colonisation de l'Algérie : à la fin de 1835 Clauzel avait demandé l'envoi de condamnés napolitains, qui auraient obtenu des concessions de terres agricoles (ASN, AE, 2381 : lettres des 14.11.1835 et 21.2.1836).

73 ASN, AE, 7053: le Prince de Scilla à d'Errico, 23.6.1841 (cf. A. Bozzo, op. cit., p. 269). Malgré cela, des napolitains avaient eu des concessions de terres dans le cadre de la colonisation promue par Bugeaud: en 1845 un tel Francesco Marini « sujet de Sa Majesté », faisait savoir d'avoir obtenu une concession de 1.80o hectares de terrain à « Group » [El-Kroub?], entre Constantine et Bône, et il demandait l'autorisation nécessaire à y faire venir 150 familles du Royaume (ASN, AE, 2385 : lettre du 9.10.1845). 
Entre temps Bugeaud avait organisé un corps d'expédition très important pour l'attaque définitive contre Abd el-Kader : l'un de ses buts était la prise et la destruction de sa capitale, Tagdempt. Cette ville, où résidaient à l'époque la famille de l'émir ainsi que celles de ses principaux collaborateurs, faisait partie d'une série de nouveaux établissements fortifiés fondés par Abd el-Kader. C'est ainsi que D'Errico les décrit dans l'un de ses rapports à Naples :

Puisque la prise de Constantine l'avait convaincu qu'aucune fortification ne pouvait résister aux armes des Chrétiens, il [Abd el-Kader] choisit pour les villes nouvelles les régions plus lointaines de l'intérieur, qu'il jugeait inaccessibles aux armées françaises chargées d'un immense matériel. Les nouveaux établissements qu'il a fondé sont Boghar, Thaga, Soida, Tafraoua, Tagdemt. Tagdemt a été fondé en 1835 par l'Émir luimême, et il est défendu par un mur qui l'encercle. Dans la ville l'Émir a réuni toutes ses manufactures, spécialement celle pour la fabrication des armes. Le fort sert comme dépôt pour les provisions de bouche et de guerre, et en plus il héberge l'hôtel de la monnaie ${ }^{74}$.

En prévoyant l'attaque française, l'émir avait éloigné vers le sud les habitants de la ville, et après le départ du corps expéditionnaire d'Alger avait fait lancer des attaques contre les fermes de ses alentours, qui étaient désormais sans défense. À Alger on avait craint des «Vêpres siciliens » 75 suite aux attaques arabes et l'état d'insécurité amenait le consul napolitain à des considérations pessimistes quant au succès de l'expédition de Bugeaud, et plus en général sur la colonisation française:

Il faut reconnaître que les Français, très habiles lorsqu'il s'agit de conquérir, sont essentiellement incapables de mettre leurs racines dans les pays conquis à cause de leur manie de réduire brusquement et sans aucune transition les choses et les hommes aux formes et aux modes de leur France. C'est un mauvais système, le contraire de ce que

74 ASN, AE, 2384: lettre du 13.4.1841. Les villes citées dans cette lettre, parfois avec des faux noms, étaient situées entre le Tell et les Hauts plateaux : Boghar à presque cent kilomètres au sud de Médéa, Taza à 65 au sud-est de Miliana, Saida à 74 au sud-est de Mascara, Tafraout à 38 au sud-ouest de Tlemcen, Tagdempt à 12 à l'ouest de Tiaret (cf. Ch.-A. Julien, op. cit., p. 184-185).

75 ASN, AE, 2384 : lettre du 14.5.1841. 
l'ancienne Reine du monde, Rome, pratiquait ; et aussi de ce que pratiquent les puissances modernes, l'Angleterre et la Russie, qui dans leurs conquêtes ont un grand respect pour les lois et les usages des peuples conquis. Elles pensent que les nouveautés, mêmes les plus évidemment salutaires, doivent s'affirmer avec le temps, avec l'exemple, avec la persuasion. C'est tout le contraire de ce que la France pratique. La furie, la légèreté, le peu de considération des Français gâche tout, et de tels agissements ne peuvent qu'engendrer la haine et la contrariété parmi les populations, spécialement lorsqu'elles n'ont aucun point de contact avec la civilisation européenne, et qu'elles sont minées par le fanatisme religieux ${ }^{76}$.

Après ces considérations, qui le voyaient convaincu de la bonté de l'indirect rule britannique, D'Errico en arrivait à conclure (peut-être à souhaiter?) que la France aurait tôt ou tard abandonné l'Algérie, après «l'avoir abreuvée de son sang et d'y avoir semé son or ${ }^{77}$.

Bugeaud était rentré â Alger quelques temps plus tard, après la destruction de la forteresse de Tagdempt et l'occupation de Mascara, mais on pouvait affirmer qu'Abd el-Kader n'avait pas subi d'irréparables pertes lors de cette expédition. Il s'était borné à évacuer les villes qu'ils ne pouvait pas défendre, sans y laisser rien d'utile, et à laisser avancer les troupes ennemies sans se battre, en assistant de loin à la souffrance des soldats français harassés par de longues marches ${ }^{78}$.

La suite de la guerre avait toutefois vu empirer la situation militaire d'Abd el-Kader, qui avait été graduellement acculé à la perte progressive des territoires sous son contrôle. Au début de 1842 le vice-consul napolitain à Oran avait envoyé un rapport sur l'abandon du champ de l'émir par plusieurs tribus de la région occidentale: on y affirmait que la raison de cela ne résidait pas dans «les sympathies de ces populations pour la domination française, mais [que c'était] l'effet de la nécessité et de l'or de la France $\gg^{79}$.

76 ASN, AE, 2384 : lettre du 18.5.1841. Dans une lettre précédente, le même consul avait affirmé, à propos d'une expédition qui se préparait contre l'émir, que même en cas de victoire la France se serait trouvé à avoir dépense une grande quantité d'argent pour une colonie qui ne lui aurait pas rapporté grandchose (ibi : lettre du 28-2-1840 ; cf. A. Bozzo, op. cit., p. 137).

77 Ibidem.

78 ASN, AE, 2384 : lettres du 15.6.1841.

79 ASN, AE, 2384 : lettre du 12.1.1842. 
On affirmait que l'émir n'avait désormais qu'une seule voie de salut, se réfugier dans le territoire marocain : en effet, l'armée française avait pris Tlemcen à nouveau, et cela avait coûté entre autres à Abd el-Kader la perte de sept canons, qui avaient été transportés et exposés à Alger ${ }^{80}$. L'émir avait essayé une contre-offensive grâce è des renforts que sûrement lui avaient été envoyés du Maroc ${ }^{81}$, mais la supériorité de l'ennemi était énorme et au fil des mois il avait perdu progressivement l'appui d'une bonne partie de ses tribus, tandis que les colonnes de Bugeaud le harcelaient en le contraignant à se déplacer sans cesse avec sa smala et les forces qui lui restaient. Au mois de mai un escadron de chevalerie commandée par le duc d'Aumale avait attaqué par surprise la smala « près de la source de Tagain », faisant une grande quantité de prisonniers et un butin important : l'exploit avait été célébré à Alger, où le gouverneur avait fait afficher dans les rues un communiqué relatant l'événement que le consul de Naples s'était empressé d'envoyer à son ministre ${ }^{82}$.

Ce revers, très grave pour son prestige, ne semblait pas avoir définitivement affaibli l'émir, qui quelques jours plus tard pouvait compter comme une victoire la mort de l'un de ses grands ennemis, le qaid Mustafa, au service de la France, tué dans un combat avec trois autres chefs collaborateurs. Cet événement, ainsi que d'autres fait d'armes où un colonel avait été tué et quelques officiers français blessés, démentaient les rapports envoyé par Bugeaud, dans lesquels on affirmait que son rival était à bout. Au contraire, affirmait le consul napolitain,

cet homme [Abd el-Kader] d'une énergie extraordinaire a encore une force morale parmi ces populations et la France devra encore supporter beaucoup d'autres sacrifices avant d'obtenir la pacification de ses possessions ici, à moins qu'elle n'ait pas la fortune d'avoir entre les mains ce nouveau Jugurtha ${ }^{83}$.

80 ASN, AE, 2384: lettre du 27.2.1842.

81 ASN, AE, 2384: lettre du 30.3.1842.

82 ASN, AE, 2384: lettre du 23.5.1843.

83 ASN, AE, 2385 : lettre du 30.5.1843. Dans plusieurs autres occasions les événements du champ de bataille avaient contredit les affirmations du gouvernement général quant au total déclin de la puissance militaire de l'émir (cf. par exemple ASN, AE, 2384 : lettre du 30.3.1842, sur la défaite du général Bédeau, qui s'est retiré « avec de sensibles pertes » après une rencontre avec « 4.000 arabes sous le commandement d'Abd el-Kader » non loin de Tlemcen). 
La police coloniale faisait entre temps son travail dans les lignes arrières : le mufti d'Alger, accusé de relations secrètes avec l'émir, avait été arrêté et envoyé en France ${ }^{84}$.

Au début du mois de septembre on signalait l'arrivée à Alger d'un navire anglais avec à son bord « un nommé James Scott » qui avait affirmé d'avoir été envoyé par Abd el-Kader pour traiter la paix, mais le gouverneur général avait refusé de le rencontrer ${ }^{85}$. La parole restait uniquement aux armes, qui étaient de plus en plus défavorables à l'émir : à la fin de 1843, il fut obligé de se réfugier au Maroc où le sultan lui accorda l'asile.

Pour le reste des vicissitudes d'Abd el-Kader les documents napolitains sont assez discontinus et ne rapportent que des bribes d'informations, par ailleurs très indirectes.

Un rapport d'octobre 1845 affirme que l'émir s'est réfugié à nouveau au Maroc, où son autorité religieuse est toujours très forte ${ }^{86}$. Le consulat napolitains parait imparfaitement au courant des accords entre la France et le Maroc, qui avec le traité de Tanger (10 septembre 1844) avaient déclaré l'émir hors-la-loi dans tous les territoires du Maroc et de l'Algérie, lorsqu'il affirme simplement dans ce rapport que le sultan « aurait assumé un engagement avec la France contre Abd el-Kader » ${ }^{87}$ : et pourtant D'Errico lors de la signature du traité avait envoyé à Naples un autre rapport dans lequel il soulignait le mécontentement de Bugeaud, qui prétendait que les plénipotentiaires français devaient à cette occasion exiger l'emprisonnement d'Abd el-Kader et sa consignation à la France ${ }^{88}$.

Les événement liés à la fuite de l'émir au Maroc et à ses conséquences sur les rapports franco-marocains, qui étaient évidemment hors de la portée des informateurs du consulat d'Alger, sont reportés épisodiquement

84 Ibidem. Presque deux ans auparavant, un autre homme de religion, chrétien cette fois, avait encouru les ires du gouverneur général pour avoir eu des rapports non autorisé avec son ennemi : l'évêque catholique Dupuch. Celui-ci avait organisé au mois de mai 1841un échange de prisonnier (150 arabes, dont un officier des réguliers d'Abd el-Kader, contre 128 européens, dont deux corallari napolitains capturés après le naufrage de leur barque le long de la côte de Cherchell), et par la suite avait continué à entretenir des rapports avec le camp ennemi malgré l'avis contraire de Bugeaud: il avait été expulsé d'Alger et renvoyé en France au mois d'août de la même année (ASN. AE, 2383 : lettres des 24.5. et 10.8.1841).

85 ASN, AE, 2384 : lettre du 2.9.1843. Les autorités françaises lui avaient prohibé de descendre du bateau.

86 ASN, AE, 2385 : lettre du 19.10.1845.

87 Ibidem ; cf. aussi A. Bozzo, op. cit., p. 144.

88 ASN, AE, 2385 : lettre du 24.9.1844. 
(et parfois avec imprécision): c'est ainsi que l'on relate les tractations pour délimiter la frontière entre l'Algérie et le Maroc, le bombardement de Tanger et de «Magadan » [Mogador] par une escadre française, la rencontre de Bugeaud et de «l'empereur du Maroc » à la frontière entre les deux pays, l'emprisonnement d'Abd el-Kader par les hommes du sultan ... ${ }^{89}$

Très peu de rapports du consulat napolitains citent par la suite Abd elKader : au mois de mars 1846 on relate l'exploit du général Yousouf, qui a surpris son champ en lui infligeant une grave défaite et en contraignant à la fuite «l'ex-Émir ${ }^{90}$. Au mois de décembre 1847 quelques nouvelles alarmantes sur la situation du Maroc arrivent à Naples : on dit qu'Abd el-Kader, avec l'intention de soulever les marocains contre le sultan, accusé d'avoir trahi l'islam et de s'être rendu à la France, s'est emparé de Taza avec 10.000 hommes armés et qu'il se dirige sur Fès ${ }^{91}$. Quelques jours plus tard, par contre, la situation d'Abd el-Kader apparaît « réellement désespérée » : il serait entré en pourparlers avec des émissaires du sultan et il aurait affirmé d'être prêt à renvoyer ses hommes et à se retirer de la lutte, en s'exilant à l'intérieur du pays ${ }^{92}$. Toutefois, les pourparlers ne durent pas longtemps: la situation reste incertaine, tandis qu'une partie de l'armée marocaine serait disposé à se révolter pour appuyer Abd el-Kader ${ }^{93}$.

Enfin, on annonce la reddition d'Abd el-Kader : « Vaincu par les troupes marocaines et serré par celles du général La Moricière, il n'avait pas trouvé d'autre solution que [de recourir à] la générosité de la France ${ }^{94}$. Il a demandé d'avoir la permission de partir au Levant, mais à ce propos le consul D'Errico doute que le gouvernement français le lui permettra.

La nouvelle de la reddition de cet ennemi redoutable est arrivée par télégraphe à Alger, où elle a été annoncée à la population par plusieurs salves de canon ${ }^{95}$.

5. En conclusion, on peut dire que les documents des archives napolitaines concernant l'épopée de l'émir Abd e-Kader, bien que n'apportant pas de nouveaux éléments ou de renseignements bouleversants quant à

89 ASN, AE, 7053 : lettres des 13.8 et 16.9.1844, 10.7.1845.

90 ASN, AE, 2385 : lettre du 30.3.1846.

91 ASN, AE, 2385 : lettre du 8.9.1847.

92 ASN, AE, 2385 : lettre du 4.12.1847.

93 ASN, AE, 2385: lettre du 18.12.1847.

94 ASN, AE, 2386 : lettre du 27.12.1847.

95 Ibidem. En pièce-jointe, copie de «L'Akbar » du 21.12.1847. 
l'histoire de cette période de l'histoire de l'Algérie, offrent aux chercheurs de nombreux points d'intérêt.

D'un côté, ils nous renseignent sur l'activité de nombreux sujets des états italiens qui agissaient à cette époque dans le territoire que la France était en train de conquérir : tels, les principaux représentants de la 'nation juive', qui étaient souvent d'origine livournaise et sujets du Grand-Duc de Toscane; les soldats engagés dans la Légion étrangère, souvent après avoir déserté et fui l'Italie au cours des instabilités politiques du Risorgimento, et qui se battaient contre les troupes de l'émir ; les corailleurs napolitains qui continuaient leur travail le long de la côte algérienne, et qui parfois, même sans le vouloir, se trouvaient participer aux vicissitudes de la guerre et de la paix; les commerçants et travailleurs qui avaient été appelés par l'émir pour l'aider dans son combat ... Parmi ces derniers, la figure la plus intéressante nous semble celle de l'oukil Garavini, qui avait accepté d'assumer une tâche qui devait lui coûter la continuité de ses affaires et de sa vie en Algérie: on aurait aimé en savoir plus sur ce personnage par les documents napolitains, mais les renseignements que ces derniers nous encouragent à un approfondissement de la recherche sur l'entourage étranger de l'émir, dont beaucoup d'éléments restent encore aujourd'hui inconnus.

Un autre élément d'intérêt est constitué par les commentaires concernant la politique coloniale et les différentes phases de la confrontation entre Abd el-Kader et la France. Au-delà des considérations personnelles des consuls napolitains, observateurs extérieurs et non impliqués dans les événements d'Algérie pour des raisons d'appartenance nationale et qui relèvent d'un débat qui devait être courant dans le milieu consulaire d'Alger, on trouve dans ces documents aussi quelques éléments inédits : par exemple, à propos de l'offre de Bugeaud (qui ne me semble pas être connue par aucune autre source) de céder quelques places côtières à l'état napolitain en échange de troupes auxiliaires. Il s'agit d'un épisode difficilement explicable avec les peu d'éléments offerts par les documents napolitains: révélation d'une difficulté réelle dans la confrontation militaire contre Abd el-Kader et de la nécessité de trouver ailleurs de quoi augmenter le poids de l'armée française ? Coup de tête d'un militaire désirant affirmer sa liberté d'action face à son gouvernement - pourquoi le gouvernement français n'aurait-il pas engagé directement des pourparlers avec l'ambassade napolitaine à Paris, si la proposition avait une base politique réfléchie - ? Boutade d'un général-colonisateur considérant que la population de son pays n'avait pas, du point de vue démographique, les moyens de réaliser une colonisation que d'autres peuples (les italiens, les espagnols...) auraient 
dû réaliser pour son compte ? Essai d'engager le royaume de Naples à côté de la France, en le détachant de la liaison, par ailleurs très stricte, avec l'Angleterre ? Ou bien clin d'oeil à la Grande Bretagne par l'entremise des napolitains, en ouvrant une perspective de 'partage' dans une région convoitée de la Méditerranée qui avait vu la puissance anglaise opposée à l'établissement d'un pouvoir français durable en Algérie?... Dans ce cas, aussi, les documents napolitains posent des questions auxquelles ils ne répondent pas, laissant au chercheur l'envie de fouiller encore.

Il me semble que l'on peut souligner — autre point d'intérêt — la quantité de 'on dit' qui parsèment la correspondance dirigée au gouvernement napolitain. Indice des bruits qui couraient à l'intérieur du pays sur tel ou tel événement, sur tel ou tel aspect de la lutte entre Abd el-Kader et ses ennemis, faute d'une connaissance précise des faits, ou bien, parfois, remise en question des vérités officielles que les autorités françaises diffusaient ? Mais aussi, indice des sentiments de la population européenne, des débats qui devaient naître parmi le personnel des consulats d'Alger, ainsi que du 'passage de renseignements réservés' provenant des capitales européennes que devaient filtrer lors de ces débats.

Je crois que cet aperçu des documents des archives napolitaines, qui aident à la compréhension d'un chapitre fondamental de l'histoire de l'Algérie au XIXe siècle, pourra servir d'impulsion à l'approfondissement de la recherche : vraisemblablement, d'autres sources doivent exister, non seulement à Naples, mais aussi dans les autres capitales des anciens états italiens qui avaient des consulat dans les villes du Maghreb à l'époque du long combat de l'émir Abd el-Kader.

\section{Appendice}

Le premier document des Archives de Naples sur l'émir Abd el-Kader dans son texte italien:

[Lettre du vice-consul napolitain à Oran, Carlo Becciani, au Ministre des Affaires étrangères du Royaume des Deux Siciles, 10.3.1834 (Archives d'État de Naples, Affaires Étrangères, 2382)]

L'avvenimento straordinario, e da tutti inaspettato quale è stato quello della pace successa tra i Francesi e i Beduini dell'interno, gente superstiziosa nemica acerrima dei Cristiani, m'arriva e m'incoraggia a dare parte a 
V.E. [...]. Un certo Sig. Moise Busnach nativo e suddito toscano già da qualche tempo pratico sia del Paese, che dei costumi degli Arabi, decorato della Legione d'Onore e amico del Signor Maresciallo Clausell è stato il promotore e l'organo principale di questa pace. Dopo varie lettere scritte, ottenuta una risposta lusinghevole dal Bey Berkader, capo supremo e condottiere potente degli Arabi, il detto Signor Busnach accompagnato da un comandante francese e dal Signor Amar capo della nazione Israelita d'Orano, partì il 27 febbraio caduto con due arabi di scorta alla volta del villaggio di Abrahah [?] dove erano attesi dal suddetto capo Berkader.

Giunsero al suddetto villaggio dopo aver percorso venti leghe di un terreno stato più volte bagnato dal sangue Francese e Beduino camino pericoloso per la sicurezza, e che venti giorni indietro sarebbe stato teatro di distruzione per chi osato avesse percorrerlo. Giunti alla presenza di Berkader furono gli ambasciatori accolti con tutti gli onori militari propri dell'Araba Nazione : musica barbara e salve di moschetteria li salutarono : colà trovarono cinque prigionieri Francesi che Berkader aveva fatto a se venire per renderli alle loro Bandiere, qualunque fosse stato l'esito delle trattative, e ciò per provare la stima che esso dice nutrire per questo Generale Capo Signor Baron Desmichels.

Dopo una dimora di quattro giorni che gli ambasciatori Francesi fanno con Berkader continuamente ricolmi d'onori e d'attenzioni, e quando si disponevano a tornare ad Orano, Berkader gli pregò di volerlo accompagnare fino al villaggio di Segh cinque leghe dal primo distante. In questa piccola traversata il seguito di Berkader forte allora di circa 200 uomini si aumentò fino a seimila cavalieri. Questa numerosa scorta era accompagnata da varie bande militari che coi loro barbari strumenti alternavano delle marcie con spesse scariche di moschetteria in segno di gioia. Giunti a Segh gli uni si congedarono dall'altro, e presero la volta di Orano unitamente a Milùd Ben Arasch Agà di Berkader che esso ha già mandato come plenipotenziario in compagnia di altri capi tribù e con scorta d'Arabi. Il cammino da Segh a poca distanza d'Orano era ingombro di Arabi cavalieri che continuamente salutavano i viandanti con scariche di moschetteria.

La sera del quattro corrente i Deputati Francesi e Arabi accompagnati dai prigionieri entrarono in città in mezzo alle acclamazioni di tutta la popolazione della guarnigione che in gran numero vi si era portata ai suo incontro. La sera dopo la prima conferenza (cioè il sei corrente) vi fu gran ballo presso il generale in capo con intervento di tutte le autorità, e dei Beduini che rimasero molto sorpresi nel vedere le donne così scoperte, $\mathrm{e}$ così corteggiate pubblicamente. 
Questo ballo riuscì brillantissimo, e si prolungò a notte avanzata. Il sette vi fu gran rivista e tutte le truppe francesi riunite eseguirono una piccola guerra con soddisfazione e piacere dei detti Beduini.

Questa pace ha sorpreso tutti conoscendo l'odio delle cabile di questi Arabi e la loro superstizione religiosa. Qualunque ne sia stato lo scopo per la parte degli Arabi, vi sarà da contare e fidare sulla Fede di Berkader che è un uomo i di cui antenati hanno più volte regnato in queste provincie, e per il momento ed in questo paese viene ad acquistare ed essere interessante per il commercio seguito, poiché una delle prime condizioni fra le altre è il commercio libero per entrambe le nazioni. Berkader che è un uomo la cui potenza si estende da Orano al di là d'Algeri, e che può mettere in armi nel tempo di due giorni cinquantamila cavalieri si rende garante di tutte le uccisioni, e della salvezza dei cristiani e della loro libertà. Così potranno terminare le stragi e l'uccisioni che continuamente aveano luogo : così il paese non mancherà più di viveri, essendo stati più volte in passato privi di carne, ova, e pollami. Sarà un vantaggio utile per il commercio aprendosi così pure la comunicazione coll'Impero del Marocco [...]. 